\begin{tabular}{|c|c|c|}
\hline$\gamma$ & $\begin{array}{c}\text { European Association for the } \\
\text { Development of Renewable Energies, Environment } \\
\text { and Power Quality (EA4EPQ) }\end{array}$ & $\begin{array}{l}\text { International Conference on Renewable Energies and Power Quality } \\
\text { (ICREPQ'12) } \\
\text { Santiago de Compostela (Spain), 28th to 30th March, } 2012\end{array}$ \\
\hline
\end{tabular}

\title{
Fuzzy Logic Based Power Management Strategy for Plug-in Hybrid Electric Vehicles with Parallel Configuration
}

\author{
Hasan Alipour, Behzad Asaei, Ghias Farivar \\ School of Electrical \& Computer Engineering \\ College of Engineering \\ University of Tehran \\ P.O.Box: 14395-515 Tehran (Iran) \\ Phone:+98 021 82084936, Fax number: +98 02188633029 , \\ E-mail: hasan.alipour2006@gmail.com, basaei@ut.ac.ir, gh_farivar@hotmail.com
}

\begin{abstract}
In this paper, a new power management strategy for parallel plug-in hybrid electric vehicles is introduced. Using the proposed strategy the fuel consumption of the vehicle would be reduced. The proposed power management strategy is simple, and the required computational burden is not heavy. Hence, it can be used in real time applications. Estimating, the trip distance using GPS technology, the controller can manage the battery state of charge. The affectivity of the introduced approach is investigated through conducted simulations in ADVISOR environment. The obtained results are validated using a simple rule base power management strategy.
\end{abstract}

\section{Key words}

Fuzzy logic controller, plug-in hybrid electric vehicle, power management strategy.

\section{Introduction}

Daily increase in fossil fuel consumption caused many problems for the societies and governments. For instance, the harmful emissions of burning these fuels such as CO and $\mathrm{NO}_{\mathrm{x}}$ are threatening to public health. Besides, the excessive amount of $\mathrm{CO}_{2}$ in the atmosphere would lead to greenhouse effect and global warming. Moreover, the sources of fossil fuels are depleting.

Since the automobiles are one of the main fossil-fuel consumers, increasing their efficiency would have a great effect on reduction of fuel usage. Concerning the near future, hybridization of the automobiles seems to be the most feasible solution in order to enhance the fuel economy of conventional vehicles. In hybrid electric vehicles (HEVs), demanded power is shared between the electric and fossil fuel sources. Furthermore, some portion of braking energy can be regenerated through the electric power train. Hence, HEVs are more efficient than the conventional vehicles. However, still the main source of the energy is the fossil fuel and there no possibility for using clean and inexhaustible energy sources.
Other proposed structure for future vehicles is pure electric vehicles (EVs). These vehicles are the cleanest, but they have several disadvantages such as battery life time and limited driving distance corresponding to the available battery charge.

Plug-in hybrid electric vehicles (PHEVs) are proposed as a new generation of the HEVs. The main difference between the PHEV and HEV topologies is the bigger battery size in PHEV. A PHEV can be plugged into an external electric source and charge its on-board batteries [1]. Therefore, the advantages and disadvantages of HEVs and EVs are compromised in PHEVs.

Beside proper vehicle components sizing, power management strategy has also an important role in the efficient performance of a PHEV. Generally, these strategies are composed of pure electric (EV), charge depletion (CD), and charge sustaining (CS) modes. These three different modes are shown in Fig. 1. In the EV mode, the engine is off and the traction power is provided by the battery. Therefore, in this mode, the battery would be discharged very fast. However, it can be charged by the regenerated energy during braking intervals. In the CD mode, the engine and motor can be operated simultaneously and the battery can be charged by the engine. However, the battery state of charge (SOC) has descending trajectory. In the CS mode, the battery $S O C$ is sustained around a specific $S O C$. In this mode, the engine and battery provide the average and dynamic demanded power, respectively. In other words, in this mode the PHEV is converted to a HEV [2].

From mathematical view point, the PHEVs power management strategies are classified into rule based and optimization based strategies [2]. Rule based strategies themselves are split to deterministic and fuzzy logic control strategies. Usually, these strategies are causal and have simple mathematics. Therefore, they can be performed by low cost processors. 
A rule based strategy for a parallel PHEV with focusing on the EV and CD modes are proposed in [1]. An engine on-off strategy with considering the acceleration pedal position is proposed in [3]. A deterministic rule based strategies for serial-parallel PHEVs is proposed in [4]. This strategy attempts to optimize the engine operation. In [5], a fuzzy logic control strategy is proposed for a parallel hybrid electric public bus.

Optimization strategies generally are more complex and accurate than rule based strategies. Usually, these strategies have complex mathematics and heavy calculation burdens. Some of these strategies are noncausal and require some priori knowledge about driving cycle. Generally, in these strategies a cost function is introduced and minimized [1]. Optimization based strategies contain large spectrum of mathematical solutions such as statistical optimization, numerical optimization, equivalence cost minimization strategy (ECMS), and analytical solutions [6].

One of the most promising power management strategies is dynamic programing (DP) which lies in numerical optimization category [7]-[8]. Therefore, DP has heavy calculation burden and it is very time consuming. Hence, two scale dynamic programing solutions are proposed to discounting the mentioned problems [9]-[11].

This paper proposes a new fuzzy logic control strategy for a parallel PHEV. This strategy is applicable and has simple concepts. This strategy is simulated in the ADVISOR environment and compared with the proposed strategy in [1].

\section{Vehicle Model}

Parallel, serial and serial-parallel structures are three different topologies of PHEVs. A parallel PHEV maintains a mechanical linkage between the internal combustion engine and the wheels. In this topology, the electric motor assists the engine to provide the demanded power. The parallel topology required smaller motor than the serial structure. Moreover, the generator is not required in this topology. Structure of the assumed parallel vehicle is shown in Fig. 2. The used notations in Fig. 2, are defined in Table I.

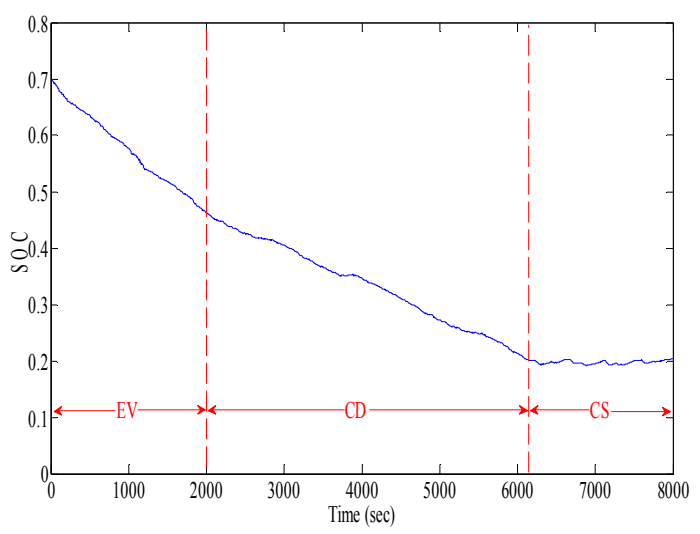

Fig. 1. Different control modes

\section{Fuzzy Logic Control Strategy}

In this paper multi input single output Mamdani's fuzzy inference method is used because of its simplicity and ease of implementation. Battery SOC and demand power are considered as the inputs and the engine power is the output. The engine efficiency map is divided to several zones in order to design the membership functions. SI41 engine efficiency map and the defined operation zones are shown in Fig. 3 . In this figure, $P_{\max }, P_{m}, P_{\max -o p t}$, and $P_{\text {min-opt }}$ are the engine maximum available power, engine optimum efficiency power, and the upper and lower limits of the efficient operation zones, respectively. It worth indicating that, these parameters are not constant and their values are dependent to speed.

Similarly, the battery $S O C$ is limited to stay in a predetermined zone as shown in Fig. 4. Concerning fuel consumption reduction, it is favourable to deplete the battery charge at the end of the trip. On the other hand, depletion of the battery charge before reaching the end of the trip would pose some limitation on the engine operation because it should provide the net power and rely on the battery only as a power buffer. Hence, in this paper, the $S O C$ reference is dependent on the distance to the end of the trip. Therefore, the trip length should be provided to the controller in advance. Knowing the destination, it is easy to estimate the trip length from GPS data. Moreover, at the beginning of the trip, the battery $S O C$ is allowed to change more freely and in a wider band. By getting closer to the end of the trip the SOC operation zone shrinks. Using this approach the controller would allow the engine to operate more freely in the optimum points and guarantee the battery charge depletion at the end of the trip.

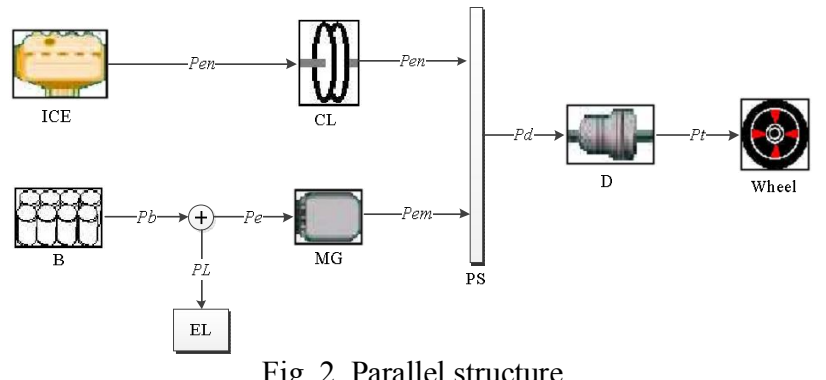

Fig. 2. Parallel structure

Table I. Notations in the vehicle model

\begin{tabular}{|l|l|}
\hline Explanation & Notation \\
\hline Internal combustion engine & ICE \\
Battery & B \\
Electrical motor / generator & MG \\
Power splitter & PS \\
Transmision & D \\
Axellary electrical load & EL \\
ICE external power & $\boldsymbol{P}_{\boldsymbol{e n}}$ \\
External battery power & $\boldsymbol{P}_{\boldsymbol{b}}$ \\
MG power in electrical side & $\boldsymbol{P}_{\boldsymbol{e}}$ \\
MG power in mechanical side & $\boldsymbol{P}_{\boldsymbol{e m}}$ \\
Elctrical axellary power & $\boldsymbol{P}_{\boldsymbol{L}}$ \\
Demanded power & $\boldsymbol{P}_{\boldsymbol{d}}$ \\
\hline
\end{tabular}


The membership functions are shown in Figs 5-7. The rules lookup table is given in Table II. The rules are designed in such a way to guarantee the efficient operation of motor and maintain the battery $S O C$ in its permitted zone. The block diagram of simulated system is given in Fig. 8.

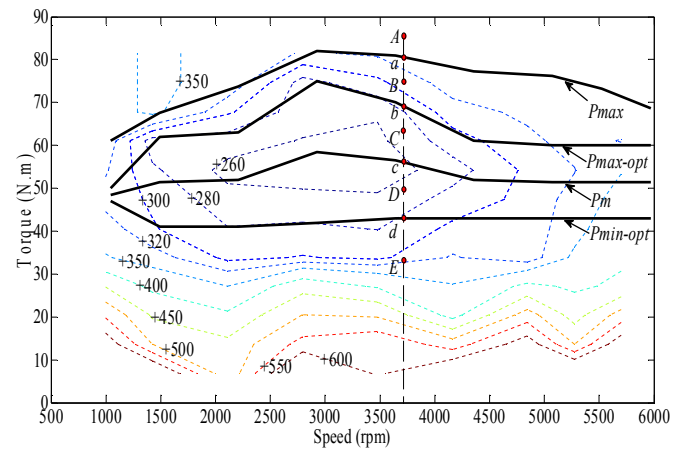

Fig. 3. The SI41 engine map ( $\mathrm{g} / \mathrm{kWh})$

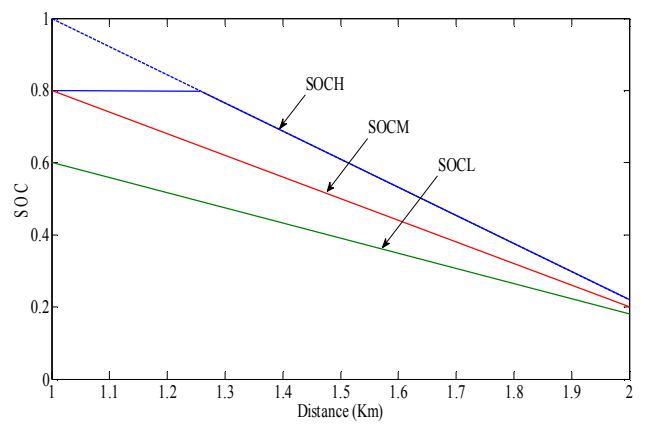

Fig. 4. Reference SOCs.

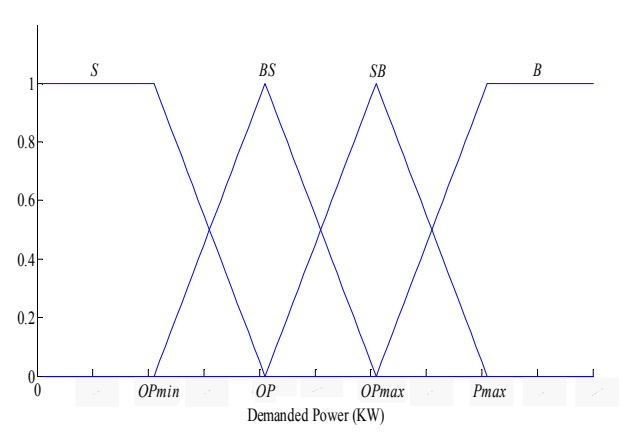

Fig. 5. Fuzzy membership function for first input (demanded power)

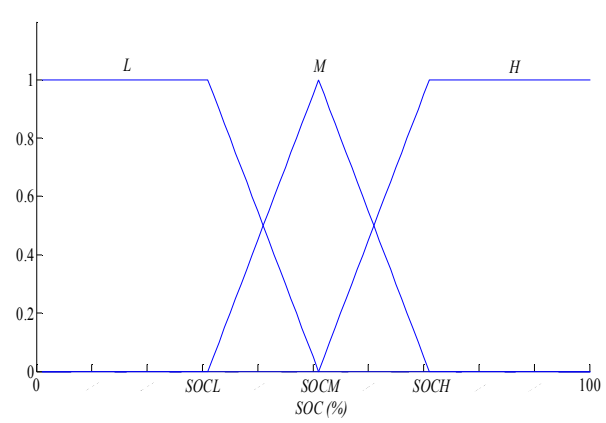

Fig. 6. Fuzzy membership function for second input (battery $S O C)$

\section{Simulation Results}

The proposed power management strategy is implemented in the ADVISOR environment. The assumed vehicle is a small city car in $\mathrm{C}$ class. Its components and parameters are given in Table III and Table IV, respectively.

Simulation results using the proposed fuzzy logic control strategy and a conventional rule based control strategy that presented in [1] are provided in table V. These simulations are done for ten repetitions of seven different driving cycles. The average fuel consumption for these cycles is used for comparison. As it can be seen, the proposed strategy is reduced the fuel consumption about $5.7 \%$.

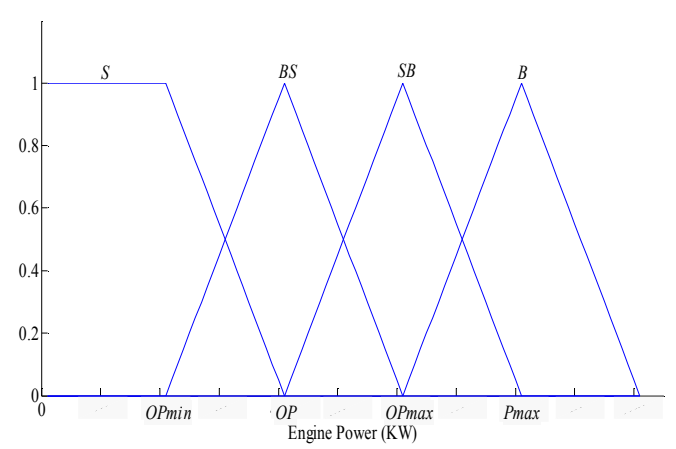

Fig. 7. Fuzzy membership function for output signal (engine power)

Table II. Fuzzy lookup table

\begin{tabular}{cccc}
\hline$P_{d} \mid S O C$ & $L$ & $M$ & $H$ \\
\hline$S$ & $B S$ & $B S$ & $S$ \\
\hline$B S$ & $S B$ & $B S$ & $B S$ \\
\hline$S B$ & $S B$ & $B S$ & $B S$ \\
\hline$B$ & $B$ & $S B$ & $B S$ \\
\hline
\end{tabular}

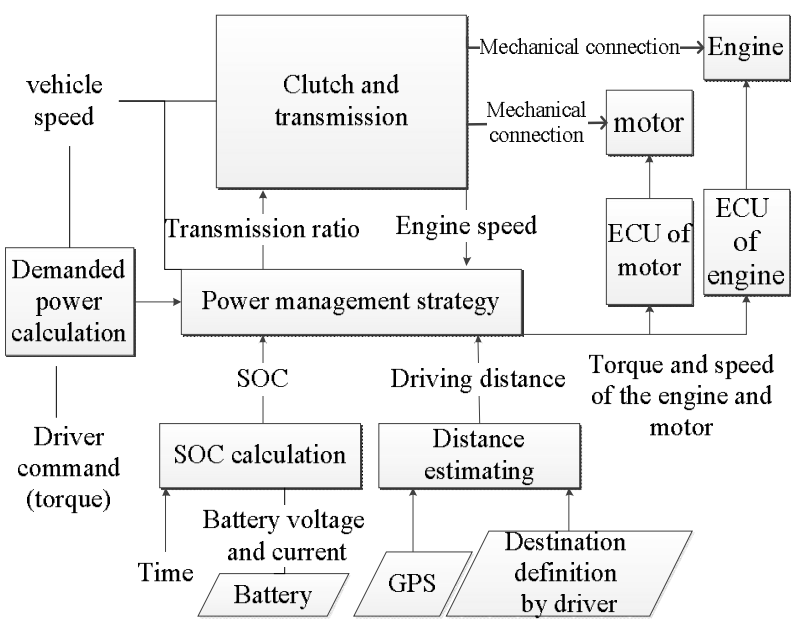

Fig. 8. Block diagram of simulated system 
The engine operation points for ten NEDC driving cycle are shown in Fig. 9. As it can be seen the ICE operating points are managed to work in the efficient areas.

The battery SOC trajectory for the same driving cycle is shown in Fig. 10. This figure reveals that the battery charge is depleted from $S O C_{H}$ to $S O C_{L}$ in the end of the trip and the $S O C$ follows the reference $S O C$ very well. As it can be seen the proposed strategy does not deplete the battery before reaching the end of the trip. Hence, it increases the controller ability to push the operating points to more efficient areas. Moreover, it guarantees the battery charge depletion at the end of the trip. Therefore it can be recharged from the grid.

Table III. The simulated vehicle components

\begin{tabular}{ll}
\hline Engine & $\begin{array}{l}\text { SI41-with 41 kW maximum power and 0.34 peak } \\
\text { efficiency }\end{array}$ \\
\hline Electric & AC59 - with $56 \mathrm{~kW}$ maximum power and 0.91 \\
machine & peak efficiency \\
Battery & Li_ion28 - with 28 Ah nominal current and 335 V \\
& nominal voltage \\
Transmission & TX_5SPD-with 1.00 peak efficiency \\
\hline
\end{tabular}

Table IV. The simulated vehicle parameters

\begin{tabular}{|c|c|c|c|}
\hline Quantity & Notation & Value & unit \\
\hline Vehicle mass & $M$ & 1400 & $K g$ \\
\hline Front area & $A_{d}$ & 2.0 & $m^{2}$ \\
\hline $\begin{array}{l}\text { Air drag } \\
\text { coefficient }\end{array}$ & $C_{d}$ & 0.3 & - \\
\hline Rolling resistance & $C_{r}$ & 0.015 & - \\
\hline Air density & $P$ & 1.2 & $\mathrm{Kg} / \mathrm{m}^{3}$ \\
\hline Gravity & $G$ & 9.8 & $\mathrm{~m} / \mathrm{s}^{2}$ \\
\hline Wheel radius & $\omega_{r}$ & 0.3 & $m$ \\
\hline Upper $S O C$ level & $S O C_{H}$ & 0.8 & - \\
\hline Lower $S O C$ level & $S O C_{L}$ & 0.2 & - \\
\hline Gear ratios & $g_{r}$ & 13.45_7.57_5.01_3.77_3.01 & - \\
\hline
\end{tabular}

Table V. Simulation results

\begin{tabular}{ccc}
\hline $\begin{array}{c}\text { Driving cycles } \\
\text { (ten } \\
\text { repetitions) }\end{array}$ & $\begin{array}{c}\text { Strategy from } \\
{[\mathbf{1}]}\end{array}$ & $\begin{array}{c}\text { Fuzzy logic } \\
\text { strategy }\end{array}$ \\
\hline EUDC & 3.1 & (Litter/100km) \\
\hline FTP & 4.4 & 2.3 \\
\hline HWFET & 3.5 & 4.2 \\
\hline India_urban & 5.4 & 3.5 \\
\hline India_hwy & 2.9 & 5.1 \\
\hline NEDC & 3.7 & 3 \\
\hline UDDS & 3.8 & 3.5 \\
\hline average & 3.83 & 3.7 \\
\hline
\end{tabular}

\section{5- Conclusion}

In this paper, a new fuzzy logic control strategy for the parallel PHEV is presented. In this strategy, the rulebased lookup table and the membership functions are designed based on the engine efficiency map. Unlike the previous methods, the proposed method decreases the battery SOC gradually. Therefore, it is more reliable than the compared strategy. Moreover, in the proposed strategy fuel consumption is $5.7 \%$ less than compared strategy. This strategy is causal and only requires the trip distance that could be obtained using GPS technology. Consequently, the proposed strategy is a powerful and applicable strategy for parallel PHEV power management.

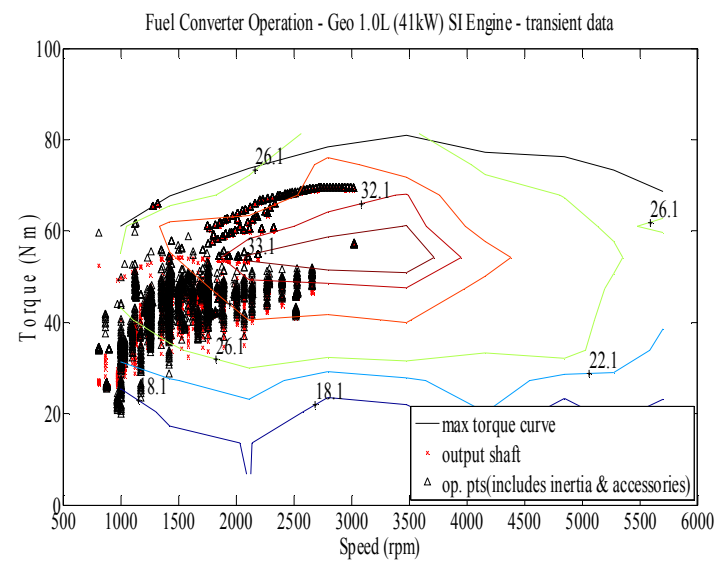

Fig. 9. Engine operating points for fuzzy logic control strategy in ten consecutive NEDC cycles

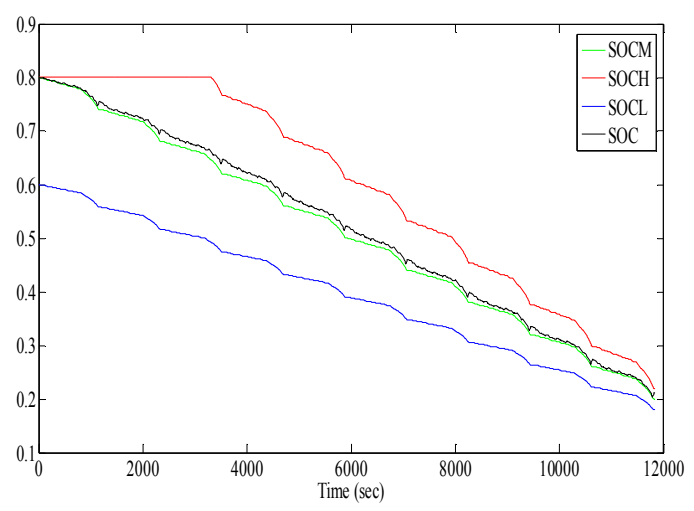

Fig. 10. SOC trajectory for fuzzy logic control strategy in ten consecutive NEDC cycles

\section{References}

[1] Yimin Gao, M. Ehsani, "Design and Control Methodology of Plug-in Hybrid Electric Vehicles," IEEE Transactions on Industrial Electronics, Vol.57, No.2, pp.633-640, Feb. 2010.

[2] S. G. Wirasingha, A. Emadi, "Classification and Review of Control Strategies for Plug-In Hybrid Electric Vehicles," IEEE Transactions on Vehicular Technology, No.99, pp.1, 0.2011. 
[3] X. Hui, D. Yunbo, "The Study of Plug-In Hybrid Electric Vehicle Power Management Strategy Simulation, "IEEE Conference on Vehicle Power and Propulsion, pp.1-3, 3-5, Sept. 2008.

[4] X. He, M. Parten, T. Maxwell, "Energy Management Strategies for a Hybrid Electric Vehicle," IEEE Conference on Vehicle Power and Propulsion, pp. 390- 394, 7-9 Sept. 2005.

[5] Li Yushan, Zeng Qingliang, Wang Chenglong, Li Yuanjie, "Research on Fuzzy Logic Control Strategy for a Plug-in Hybrid Electric City Public Bus," International Conference on Measuring Technology and Mechatronics Automation (ICMTMA), Vol.3, No., pp.88-91, 13-14 March 2010.

[6] A. Sciarretta, L. Guzzella, "Control of Hybrid Electric Vehicles," IEEE Control Systems Magazine, vol.27, no.2, pp.60-70, April 2007.

[7] Caiying Shen, Xia Chaoying, "Optimal Power Split in a Hybrid Electric Vehicle Using Improved Dynamic Programming," Power and Energy Engineering Conference (APPEEC), pp.1-4, 28-31 March 2010.

[8] Chenghong Yang, Jun Li, Wei Sun, Bo Zhang, Ying Gao, Xuefeng Yin, "Study on Global Optimization of Plug-In Hybrid Electric Vehicle Energy Management Strategies," Power and Energy Engineering Conference (APPEEC), pp.1-5, 28-31 March 2010.

[9] Qiuming Gong, Yaoyu Li, Zhong-Ren Peng, "Trip Based Power Management of Plug-in Hybrid Electric Vehicle with Two-Scale Dynamic Programming," Vehicle Power and Propulsion Conference, IEEE, pp.12-19, 9-12 Sept. 2007.

[10]Qiuming Gong, Yaoyu Li, Zhong-Ren Peng, "Computationally Efficient Optimal Power Management for Plug-in Hybrid Electric Vehicles Based on Spatial-domain Two-scale Dynamic Programming," IEEE International Conference on Vehicular Electronics and Safety, pp.90-95, 22-24 Sept. 2008.

[11]Qiuming Gong, Yaoyu Li, Zhongren Peng, "Power management of plug-in hybrid electric vehicles using neural network based trip modeling," American Control Conference, pp.4601-4606, 10-12 June 2009. 